\title{
Academic Stress Predicted by Academic Procrastination among Young Adults: Moderating Role of Peer Influence Resistance
}

\author{
Madeha Ashraf, Jamil A. Malik, Sadia Musharraf
}

\begin{abstract}
OBJECTIVE: The effect of academic procrastination in predicting academic stress among young adults. Additionally, it is aimed to explore the moderating role of peer influence resistance.

METHODOLOGY: The study used a correlations design with survey method. Data was collected from various government and private universities of Rawalpindi and Islamabad. Data was collected from December 2016 to January 2017. The sample included $N=400$ young adults including $(n=200)$ males and $(n=200)$ females with age ranging from 18 years to 25 years. Along with a demographic sheet, participants responses were collected on the Resistance to Peer Influence Scale, Academic Stress Scale and Tuckman Procrastination Scale were used. Correlation analysis was computed to investigate the relationship between academic procrastination, academic stress and peer influence resistance.

RESULTS: A significant positive relationship between academic procrastination and academic stress was evidenced whereas, significant negative relationship between peer influence resistance and academic procrastination, as well between peer influence resistance and academic stress was reported. Results also showed that females are more resistant to peer influence as compared to males whereas males appeared to procrastinate more as compared to females. Moderation analysis was showed that high levels of peer influence resistance buffered against effect of academic procrastination on to the academic stress.

CONCLUSION: It is concluded that peer influence resistance is a protective factor against negative consequences of academic procrastination in decreasing academic stress.
\end{abstract}

KEY WORDS: Young adults, Academic procrastination, Academic stress, Peer influence resistance.

This article may be cited as: Ashraf M, Malik JA, Musharraf S. Academic Stress Predicted by Academic Procrastination among Young Adults: Moderating Role of Peer Influence Resistance. J Liaquat Uni Med Health Sci. 2019;18(01):65-70. doi: 10.22442/jlumhs.191810603

\section{INTRODUCTION}

Pre-adulthood is the time when a man is most powerless to peer influence since companions turn into an essential impact on conduct during immaturity, and peer influence has been known as a trademark of pre-adult experience ${ }^{1}$. Peer congruity in youngsters is most articulated as for style, taste, appearance, belief system, and qualities ${ }^{2}$. Peer influence is normally connected with scenes of pre-adult hazard taking in light of the fact that these exercises usually happen in the organization of companions ${ }^{3}$. Connection with companions who take part in hazard practices has been appeared to be a solid indicator of a pre-adult's own conduct ${ }^{4}$.

Procrastination is the act of doing less earnest errands in inclination to more pressing ones, or accomplishing more pleasurable things set up of less pleasurable ones, and consequently putting off approaching assignments to a later time, now and again to the "latest possible time" before a due date. Procrastination is characterized as a purposive deferral of an anticipated sequence of act, in spite of being aware of undesirable results ${ }^{5}$. Dealing with stress in regular day to day existence is imperative for keeping up general wellbeing as it can enhance temperament, help resistant capacity, advance life span and permit ones to be more gainful. Academic stress is a psychological distress which might result because of projected disappointment related to academic disappointment ${ }^{6}$. Examinations, understanding the lecture, competing with other fellows, inquiries in classrooms, pleasing parents and educators by achieving their academic desires and presenting progress in subjects are some of the academic challenges which students might confront on a routine basis.

Ratsameemonthon ${ }^{7}$ suggested that last minute exam preparations and course compilation to procrastinate is a universal predisposition among university students. Nakalema and Ssenyonga ${ }^{8}$ revealed that distress and poor academic execution are due to poor time administration and last minute studying for examinations. Longitudinal studies ${ }^{9}$ give some support that stress is the result of procrastination. It means that procrastination causes stress and they are positively associated with each other. The 
phenomenon of procrastination is an extremely widespread among university students ${ }^{9}$. Procrastination was observed to be associated with maladaptive mental and academic results ${ }^{10}$. Therefore we assume that there is positive relationship between academic procrastination and academic stress and academic procrastination predicts academic stress among young adults. Furthermore, studies based on gender regarding procrastination showed that male students' procrastination is more common ${ }^{11}$. Results of Balkis, Duru $2013^{12}$ demonstrated that males are more projected to procrastinate than females. Hence we assume that male students procrastinate more as compared to female students.

A link between peer influence resistance and academic procrastination is documented in very small number of researches. A study conducted by LowingerRJ $2014^{13}$ suggested that through resistance to peer influence, self-esteem negatively related to procrastination. Peer can influence the learning and making the whole group procrastinate. Bunches with the least levels of accomplishment had a tendency to be those containing students who conveyed procrastinating most repeatedly on performing regulatory undertakings. This means that peer influence resistance and academic procrastination are negatively related ${ }^{14}$. Therefore we assume that there is negative relationship between peer influence resistance and academic procrastination.

Objective of the present study is to investigate the moderated role of peer influence resistance in predicting academic stress from academic procrastination among young adults. The present study assumes that there is negative relationship between peer influence resistance and academic stress. Another purpose of the present study is to explore the moderating role of peer influence resistance with academic procrastination and academic stress among young adults.

\section{METHODOLOGY}

The study used a correlations design with survey method. Study was approved by the institutional ethical review board. Data was collected from December 2016 to January 2017. A sample of 400 students $(N=400)$ including male $(n=200)$ and female $(n=200)$ with age range 18-25 were collected through convenience sampling from different various government and private universities of Islamabad and Rawalpindi. Informed consent was taken from each participant and they were explained about the purpose behind the research. The four scales in addition to demographic sheet were given to participants. The participants were guaranteed that the data taken from them will be kept confidential and exclusively utilized for the research purpose. Correlation analysis was computed to investigate the relationship between academic procrastination, academic stress and peer influence resistance.

\section{Instruments}

Resistance to Peer Influence: The resistance to peer influence scale was developed by Steinberg and Monahan $^{3}$. The original reliability of 10 items resistance to peer influence scale ranges from .70 to .76. Responses are coded on a 4-point scale, ranging from "Really true" for one descriptor to "Really true" for the other descriptor, and averaged. Higher scores indicate greater resistance to peer influence and vice versa.

Tuckman Procrastination Scale: The Academic Procrastination scale was developed by Tuckman $^{15}$. The original reliability of 16 items Tuckman academic procrastination scale is .86 . It is a four point rating scale. Response categories are, that's me for sure (1), that's my tendency (2), that's not me for sure (3), that's not my tendency (4). High scores show high academic procrastination and vice versa.

Academic Stress Scale: The Academic Stress scale was developed by Kohn JP $1986^{15}$. The original reliability of 35 items scale Academic Stress is .92. It is a five point rating scale. Response categories are, $1=$ not at all stressful, $2=$ rarely stressful, $3=$ sometimes stressful, 4=fairly stressful, 5=extremely stressful. A higher score is indicative of greater stress and vice versa.

\section{RESULTS}

Preliminary analysis showed that alpha reliability coefficients presented in Table II for academic procrastination scale, academic stress scale and peer influence resistance scale, appeared to be medium to good. Statistics presented in Table II shows that all variable has a normal distribution and suitable for parametric testing. Table II further displays the correlation matrix for peer influence resistance, academic procrastination, academic stress and some of the demographic variables. This also displays means and standard deviations on study variables and some of the demographic variables. The correlation between age and academic procrastination was negative and statistically significant $(r=-.15$, $\mathrm{p}<.01)$. The correlation between peer influence resistance and academic procrastination was negative and statistically significant $(r=-.50, p<.01)$. The correlation between peer influence resistance and academic stress was negative and statistically significant $(r=-.35, p<.01)$. The correlation between academic procrastination and academic stress was positive and statistically significant $(r=.33, p<.01)$. Gender differences were tested using independent 
sample t-test. Results presented in Table III showed a significant deference for peer influence resistance. An examination of the mean for peer influence resistance revealed that females were more resistant to peer influence (Mean difference $=2.97, p<.01$ ) than their males counterparts. Further results showed that male appeared to procrastinate more (Mean difference $=$ $2.67, p<.01)$ than females. No significant difference appeared on academic stress across gender.

Finally, moderation was explored using Process Macro in SPSS 21 to see the effect of peer influence resistance in predicting academic stress from academic procrastination. Table IV depicts results of moderation analysis while controlling the effect of age, gender, domain, residence (hostel versus home), and monthly family income. As was expected, academic procrastination positively predicted academic stress of young adults $(B=.52, p<.01)$ suggesting that procrastination increases stress whereas resistance to peer influence negatively predicted academic stress $(B=1.08, p<.01)$ suggesting that peer who successfully resist to peer influence faces low academic stress. Further, a significant interaction term indicates that peer influence resistance moderated the relationship between academic procrastination and academic stress. The moderating effect of peer influence resistance is elaborated in modgraph presented in Figure I. The figure suggests that academic procrastination increases and academic stress are positively associated under low to medium levels of peer influence resistance yet under high levels of peer influence resistance the academic procrastination has no more negative consequences in accumulation of academic stress.

\section{TABLE I: DEMOGRAPHIC PROFILE OF THE} SAMPLE $(n=400)$

\begin{tabular}{|c|c|c|c|}
\hline Demographic & Groups & $\mathbf{F}$ & $\%$ \\
\hline \multirow{2}{*}{ Gender } & Male & 200 & 50 \\
\hline & Female & 200 & 50 \\
\hline \multirow{3}{*}{ Program } & BS & 239 & 59.8 \\
\hline & MS/MSc & 120 & 30 \\
\hline & MPhil & 41 & 10.2 \\
\hline \multirow{2}{*}{ Domain } & Natural Sciences & 172 & 43 \\
\hline & Social Sciences & 228 & 57 \\
\hline \multirow{2}{*}{ Residence } & Hostel & 144 & 36 \\
\hline & Home & 256 & 64 \\
\hline \multirow{3}{*}{ Family Size } & Small ( $\leq 4$ member) & 81 & 20.3 \\
\hline & Medium (5-6 members) & 169 & 42.2 \\
\hline & Large (> 6 members) & 146 & 36.5 \\
\hline
\end{tabular}

TABLE II: PEARSON BIVARIATE CORRELATION AMONG STUDY VARIABLES $(n=400)$

\begin{tabular}{|c|l|c|c|c|c|c|c|c|}
\hline S. No. & Variables & No of Items & $\mathbf{\alpha}$ & $\mathbf{1}$ & $\mathbf{2}$ & $\mathbf{3}$ & $\mathbf{4}$ & $\mathbf{5}$ \\
\hline 1 & Age & - & - & - & -.03 & .08 & $-.15^{\star *}$ & -.07 \\
\hline 2 & Income (In 10K) & - & - & - & - & -.07 & .08 & .02 \\
\hline 3 & Peer Influence Resistance & 10 & .66 & - & - & - & $-.50^{* *}$ & $-.35^{* *}$ \\
\hline 4 & Academic Procrastination & 16 & .81 & - & - & - & - & $.33^{* *}$ \\
\hline 5 & Academic Stress & 35 & .89 & - & - & - & - & - \\
\hline & $M$ & - & - & 21.16 & 11.136 & 23.95 & 25.22 & 98.68 \\
\hline & SD & - & 1.88 & 24.31 & 5.32 & 8.77 & 21.84 \\
\hline${ }^{*} p<.05,{ }^{* *} p<.01, \mathrm{M}=$ Mean; SD = Standard Deviation
\end{tabular}

TABLE III: MEAN DIFFERENCES IN STUDY VARIABLES ACROSS GENDER $(n=400)$

\begin{tabular}{|c|c|c|c|c|c|c|c|c|c|}
\hline \multirow{2}{*}{ Variables } & \multicolumn{2}{|c|}{ Male $(n=200)$} & \multicolumn{2}{|c|}{ Female $(n=200)$} & \multirow{2}{*}{$T(398)$} & \multirow{2}{*}{$p$} & \multicolumn{2}{|c|}{$95 \% \mathrm{Cl}$} & \multirow{2}{*}{ Cohen's d } \\
\hline & $M$ & $S D$ & $M$ & $S D$ & & & $L L$ & $U L$ & \\
\hline Age & 21.42 & 1.99 & 20.90 & 1.71 & 2.76 & .00 & .15 & .88 & .28 \\
\hline Income & 13.03 & 32.17 & 92.37 & 11.93 & 1.56 & .00 & -97.27 & 85.68 & .16 \\
\hline Peer Influence Resistance & 22.47 & 5.83 & 25.44 & 4.29 & -5.80 & .00 & -3.97 & -1.96 & .58 \\
\hline Academic Procrastination & 26.56 & 9.36 & 23.89 & 7.93 & 3.08 & .00 & .96 & 4.38 & .30 \\
\hline Academic Stress & 100.05 & 22.87 & 97.32 & 20.73 & 1.25 & .21 & -1.56 & 7.02 & .12 \\
\hline
\end{tabular}


Stress \& Procrastination, Moderation by PIR

TABLE IV:

REGRESSION ANALYSIS FOR TESTING MODERATION BY PEER INFLUENCE resistance $(\mathrm{n}=400)$

\begin{tabular}{|c|c|c|c|c|}
\hline \multirow{4}{*}{ Predictor } & \multicolumn{4}{|c|}{ Academic Stress } \\
\hline & \multirow[b]{3}{*}{ Model $1 \mathrm{~B}$} & \multirow[b]{3}{*}{ B } & \multirow{2}{*}{\multicolumn{2}{|c|}{$\frac{\text { Model } 2}{95 \% \mathrm{Cl}}$}} \\
\hline & & & & \\
\hline & & & $L L$ & $U L$ \\
\hline Constant & $117.72^{\star *}$ & $95.81^{* *}$ & 70.79 & 120.83 \\
\hline Age & -.08 & .12 & -.93 & 1.18 \\
\hline Gender & 1.38 & 3.81 & -.58 & 8.22 \\
\hline Domain & $-4.66^{*}$ & $-4.52^{* *}$ & -8.44 & -.60 \\
\hline Residence & .60 & -.26 & -4.54 & 4.01 \\
\hline Monthly Family Income & -4.65 & .00 & .00 & .00 \\
\hline Peer Influence Resistance & $-1.08^{\star *}$ & $-.88^{\star *}$ & -1.33 & -.45 \\
\hline Academic Procrastination & $.52^{\star *}$ & $.49^{* *}$ & .23 & .75 \\
\hline Peer Influence Resistance $\times$ Academic Procrastination & & $-.09^{* *}$ & -.13 & -.06 \\
\hline$R^{2}$ & .17 & & & \\
\hline$\Delta R^{2}$ & & & & \\
\hline$F$ & $11.7^{\star *}$ & & & \\
\hline$\Delta F$ & & & & \\
\hline
\end{tabular}

FIGURE I: GRAPH SHOWING MODERATING EFFECT OF PEER INFLUENCE RESISTANCE BETWEEN ACADEMIC PROCRASTINATION AND ACADEMIC STRESS

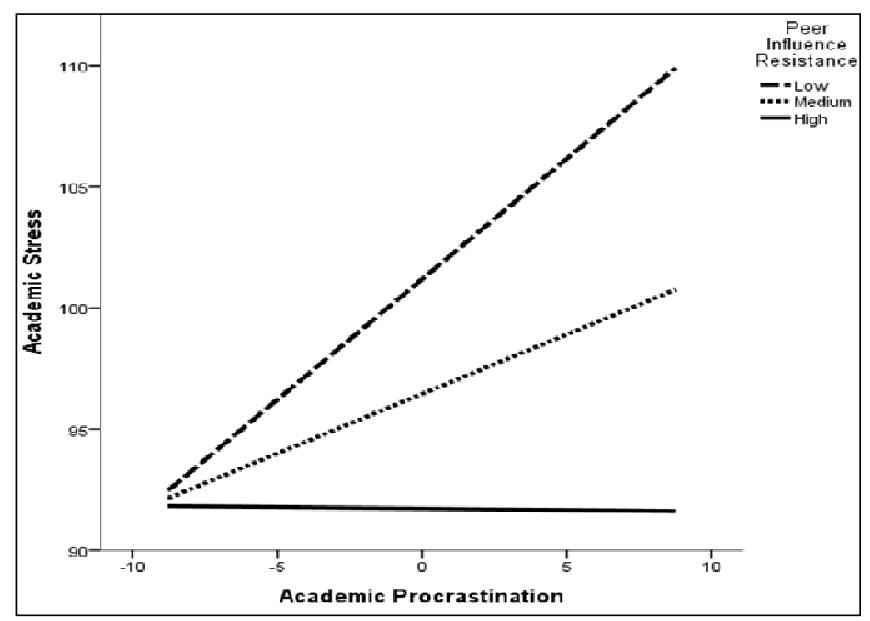

\section{DISCUSSION}

Starting with descriptive analyses and testing of assumptions for parametric statistic, the results of academic procrastination, academic stress and peer influence resistance scale showed evidences supporting continuation of hypotheses testing for the study. The scales had skewnesss and kurtosis values within \pm 1 indicating that data has a normal distribution in the sample and hence sample is assumed to be representative of population ${ }^{16}$. Among demographic variables, non-normal distribution of family income is again a true representation of population given that income has a skewed distribution with a small number of people having very high income compare to a large population on a relatively low income.

Correlation results supported our assumptions showing a negative relationship between peer influence resistance and academic procrastination. These results are also supported with earlier empirical investigations suggesting that students with elevated self-regard may probably oppose the influence of their peers, which may add to a decline in their level of lingering ${ }^{17}$. These results stress that peer relations might have a vital role in comprehending procrastination among students. Young adults with low levels of self-regard, effectively keeping up their associations with peers embrace some significant pitfalls. That is, low self-regard may propel people to yield an act (e.g., errand postponement) to reestablish social enclosure to acceptable stages. As a result, when confronted with psychosocial components, for example, peer influence and stress, the dawdling of 
growing young adults with low self-regard may mirror the results of their tradeoff between endeavors put resources into looking for peer insertion and endeavors put resources into finishing one's own errand. This trade off resulting in a negative relationship between peer influence resistance and their academic procrastination ${ }^{18}$.

Further, the results of the study also supported our assumption regarding significant negative relationship between peer influence resistance and academic stress. As resistance to peer influence increases academic stress decreases. Literature showing that peer influence resistance and academic procrastination are negatively related with each other. Study suggests that amenable gatherings cost large amount of procrastination and in academic settings assignment aversiveness are largely due to their peer influence ${ }^{14}$. Additionally, procrastination is well recognized to have undesirable outcomes for mental prosperity. Pathological studies showed that procrastination is related to stress and melancholy ${ }^{19}$. Further, our results supported the assumptions stating a significant positive relationship among academic procrastination and academic stress. Among university students procrastination is a typical problem that students confronted. There are some studies appealing that procrastination causes stress, high apprehension and disease, and furthermore few researchers indicate that procrastination is adversely linked with self-regard. This study uncovered that conduct of procrastination is anticipated through academic inspiration and essential mental needs. Hence, academic stress is predictable through academic procrastination ${ }^{20}$.

Our data suggest some gender differences that may warrant further explanations. As extensive research conducted on procrastination shows little evidence of gender differences. Our results suggesting that academic procrastination is high in men than women are in line with studies demonstrating that male students procrastinate more commonly ${ }^{11}$. Results of Balkis M 2013 ${ }^{12}$ also demonstrated that males are more projected to procrastinate than females. Additionally, as was expected female appear to have more resistance to peer influence that is no wonder given the traditional cultural role of young female in our society. The social taboos encourage young females to have a preference of staying home than accompanying peers whereas young men are encouraged to socialize and indulge in peers.

Finally, to test our assumption that peer influence resistance moderates the relationship between academic procrastination and academic stress. Results suggested that high levels of resistance to peer influence have potential to alleviate negative consequences of academic procrastination in accumulation of academic stress.

\section{CONCLUSION}

The present research evidenced that academic procrastination induced academic stress yet resistance to peer influence guard against such negative consequences. It is recommended that interventions shall be designed to boost resistance to peer influence among young adults to fight against stress induced by academic procrastination.

\section{REFERENCES}

1. Jewell JA, Brown CS. Relations among gender typicality, peer relations, and mental health during early adolescence. Social Development. 2014; 23 (1):137-156.

2. Zhang J, Tang J, Li J, Liu Y, Xing C. Who influenced you? Predicting retweet via social influence locality. ACM Transactions on Knowledge Discovery from Data. 2015; 9(3): A1-A27.

3. Steinberg L, Monahan KC. Age differences in resistance to peer influence. Dev Psychol. 2007; 43 (6):1531-1543. doi: 10.1037/0012-1649.43.6.1531

4. Ibubeleye EM, Goodness W, Chidozie IG. Self-esteem and sociological factors as correlates of alcohol consumption among university undergraduates. 2016; 7(1): 85-92.

5. Steel P, Ferrari J. Sex, Education and Procrastination: An Epidemiological study of Procrastinators' Characteristics from a Global sample. Eur J Pers. 2013; 27(1):51-58.

6. Lal K. Academic stress among adolescent in relation to intelligence and demographic factors. AIJRHASS. 2014; 5(1):123-29.

7. Ratsameemonthon L. Understanding and managing procrastination among Thai college students. Hatyai Academic J. 2015; 13(1): 75-83.

8. Nakalema G, Ssenyonga J. Academic stress: Its causes and results at a Ugandan University. African Journal of Teacher Education. 2014; 3(3): $1-21$.

9. Ozer BU, Demir A, Ferrari JR. Reducing academic procrastination through a group treatment program: A pilot study. J Rat-Emo Cognitive-Behav Ther. 2013; 31(3): 127-135. doi. 10.1007/s10942-013-0165-0

10. Sirois FM, Kitner R. Less Adaptive or More Maladaptive? A Meta-analytic Investigation of Procrastination and Coping. Eur J Personality. 2015; 29(4): 433-444.

11. Khan MJ, Arif H, Noor SS, Muneer S. Academic procrastination among male and female university and college students. FWU J Soc Sci. 2014; 8(2): 65-70. 
12. Balkis M, Duru E, Bulus M. Analysis of the relation between academic procrastination, academic rational/irrational beliefs, time preferences to study for exams, and academic achievement: a structural model. European journal of psychology of education. 2013; 28(3): 825-839. doi. 10.1007/ s10212-012-0142-5.

13. Lowinger RJ, Zhaomin H, Miranda L, Mei C. The impact of academic self-efficacy, acculturation difficulties, and language abilities on procrastination behavior in Chinese international students. College Student Journal. 2014; 48(1): 141-152.

14. Tuckman BW. The development and concurrent validity of the procrastination scale. Educ Psychol Meas. 1991; 51(2):473-480. doi.10.1177/ 0013164491512022.

15. Kohn JP, Frazer GH. An academic stress scale: Identification and rated importance of academic stressors. Psychological Reports. 1986; 59(2):415 -426. Doi. 10.2466/pr0.1986. 59.2.415

16. Schroeder L, Sjoquist D, Stephan P. Understand- ing regression analysis: An introductory guide No.57. In: Quantitative Applications in the Social Sciences. Sage Publications, Newbury Park (1986).

17. Katz I, Eilot K, Nevo N. "I'll do it later": Type of motivation, self-efficacy and homework procrastination. Motivation and Emotion. 2014; 38 (1):111-119. Doi. 10.1007/s11031-013-9366-

18. Chen BB, Shi Z, Wang Y. Do peers matter? Resistance to peer influence as a mediator between self-esteem and procrastination among undergraduates. Front Psychol. 2016; 7(12): 1-6. Doi. 10.3389/fpsyg.2016.01529

19. Sirois FM. Procrastination and stress: Exploring the role of self-compassion. Self and Identity. 2014; 13(2): 128-145. doi.10.1080/15298868. 2013.763404

20. Cavusoglu C, Karatas H. Academic Procrastination of Undergraduates: Self-determination Theory and Academic Motivation. Anthropologist. 2015; 20(3): 735-43.

\begin{tabular}{|l|}
\multicolumn{1}{c|}{ AUTHOR AFFILIATION: } \\
Madeha Ashraf \\
National Institute of Psychology \\
Quaid.i.Azam University Islamabad, Pakistan. \\
Jamil A. Malik (Corresponding Author) \\
National Institute of Psychology \\
Quaid.i.Azam University Islamabad, Pakistan \\
Email: ja.malik@nip.edu.pk \\
Sadia Musharraf \\
National Institute of Psychology \\
Quaid.i.Azam University Islamabad, Pakistan. \\
\hline
\end{tabular}

